\title{
Traumatic Dislocation of the Knee Unsuitable for the Reconstruction of Ligamentous Injuries Because of Vascular Injury: A Case Report
}

\author{
Ukai T*, Kosuke $\mathrm{H}$ and Watanabe $M$ \\ Department of Orthopaedic Surgery, Surgical Science, \\ Tokai University School of Medicine, Kanagawa, J apan \\ *Corresponding author: Ukai T, Department of \\ Orthopaedic Surgery, Surgical Science, Tokai University \\ School of Medicine, 143 Shimokasuya, Isehara, Kanagawa \\ 259-1193, J apan
}

Received: May 14, 2021; Accepted: June 14, 2021; Published: J une 21, 2021

\begin{abstract}
Traumatic dislocation of the knee is rare, but often requires surgical intervention because complications frequently occur, including various ligamentous injuries. In this study, we report a case of knee dislocation with ligamentous injuries and injury to the popliteal artery.

The patient was a 32-year-old man. He fell while bouldering and was diagnosed with dislocation of the right knee and multiple ligamentous injuries. Upon examination, we found that his right dorsal foot was numb, and the dorsal artery of the right foot was not palpable. His right leg felt cold compared to the other leg. Magnetic resonance imaging revealed injuries to the anterior cruciate ligament, posterior cruciate ligament, and lateral collateral ligament. Computed tomography revealed that the patient's right popliteal artery was partially obstructed. We planned to reconstruct the injured ligaments. Prior to the surgery, the radiologist performed angiography, and the patient's popliteal artery became completely obstructed during angiography; therefore, revascularization was performed immediately. Although the blood flow in the right leg recovered, it remained cold, and we did not reconstruct the ligamentous injuries.
\end{abstract}

Knee dislocation requiring revascularization is quite rare. However, it causes severe dysfunction of the knee. Thus, meticulous and frequent evaluation of vascular injuries is necessary for the treatment of knee dislocation.

Keywords: Case report; Knee dislocation; Vascular injury; Ligamentous injury; Ligament

\section{Abbreviations}

ACL: Anterior Cruciate Ligament; PCL: Posterior Cruciate Ligament; MCL: Medial Collateral Ligament; LCL: Lateral Collateral Ligament; TA: Tibialis Anterior; EHL: Extensor Hallucis Longus; MRI: Magnetic Resonance Imaging; CT: Computed Tomography; KD: Knee Dislocation; ABI: Ankle-Brachial Pressure Index

\section{Case Presentation}

Traumatic knee dislocation accounts for $0.02 \%-0.2 \%$ of all dislocations [1-6]. Most patients with traumatic knee dislocation also present with ligamentous injuries, such as Anterior Cruciate Ligament (ACL), Posterior Cruciate Ligament (PCL), Medial Collateral Ligament (MCL), and Lateral Collateral Ligament (LCL) injuries that require reconstruction. Reports suggest that an improved range of motion is seen in patients who undergo surgical reconstruction compared to patients managed nonoperatively, and that nonoperative treatment results in a decreased range of motion, fixed flexion deformity, and poor Lysholm scores [7-11].

Natsuhara et al. investigated 8050 cases of knee dislocations and reported that the incidence of arterial injury with knee dislocation is $3.3 \%$ [12]. Traumatic knee dislocation with arterial injury is quite rare, so there are very few reports on knee dislocation with arterial injury requiring revascularization. Arterial injury must be treated promptly and appropriately, because once the patients experience complications with a vascular injury, they may require amputation. Thus, simultaneous evaluation of ligamentous and arterial injuries is necessary in knee dislocation. In this study, we present a case of traumatic knee dislocation where reconstruction of the ligamentous injuries could not be performed due to vascular injury.

A 32-year-old man fell while bouldering and his right knee was hyper-extended. He consulted a doctor on the day of injury, and he was diagnosed with dislocation of the right knee and multiple ligamentous injuries of the knee. His right knee was fixed with a knee brace and he was referred to our hospital. According to the medical records from the previous hospital, the dorsal artery of the right foot was palpable and he scored 1 out of 5 in a manual muscle test of his Tibialis Anterior (TA) and Extensor Hallucis Longus (EHL) muscles. However, when he was admitted to our hospital, the weakness in his right foot had recovered completely. In contrast, we confirmed numbness of the right dorsal foot, and the dorsal artery of the right foot was not palpable. Compared to his left limb, his right leg and foot felt cold. Physical examination revealed positive Lachman, Pivot shift, varus stress, posterior drawer, and dial tests. Roentgenography performed at the previous hospital revealed dislocation of the right knee and dislocation of the tibia to the former side (Figure 1). Magnetic Resonance Imaging (MRI) revealed injuries to the ACL, PCL, and LCL of his right knee (Figure 2). Contrast-
Austin J Orthopade \& Rheumatol - Volume 8 Issue 1 - 2021 ISSN: 2472-369X | www.austinpublishing group.com Ukai et al. (C) All rights are reserved
Citation: Ukai T, Kosuke H and Watanabe M. Traumatic Dislocation of the Knee Unsuitable for the Reconstruction of Ligamentous Injuries Because of Vascular Injury: A Case Report. Austin J Orthopade \& Rheumatol. 2021; 8(1): 1099. 

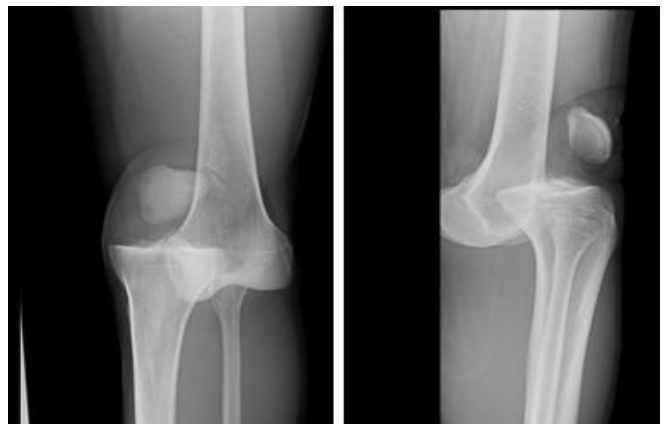

Figure 1: Radiograph showing dislocation of the right knee.

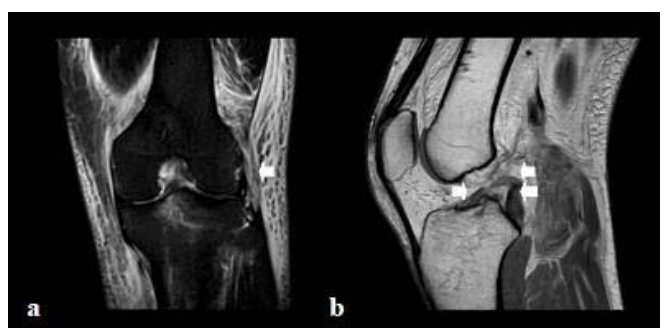

Figure 2: Magnetic resonance images. a) Coronal image showing an LCL injury (arrow); b) Sagittal image showing an ACL injury (single arrow) and a PCL injury (double arrows). ACL: Anterior Cruciate Ligament; LCL: Lateral Collateral Ligament; PCL: Posterior Cruciate Ligament.

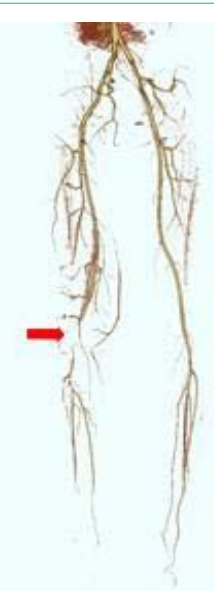

Figure 3: Contrast-enhanced computed tomography image showing interruption of the right popliteal artery (red arrow).

enhanced Computer Tomography (CT) revealed that the right popliteal artery was obstructed at the level of the knee joint (Figure 3). We diagnosed the patient with ligamentous injuries of the knee (ACL, PCL, and LCL) and popliteal artery injury. We planned to perform angiography before reconstructing the ligamentous injuries. However, blood flow in the lower leg disappeared during angiography (Figure 4) and revascularization was performed immediately using an artificial blood vessel. After the operation, the blood flow of the limb recovered; however, the right leg remained cold and we did not attempt reconstruction of the ligamentous injury because we anticipated that reconstruction of multiple ligamentous injuries needed a longer operative time and use of a tourniquet, which would

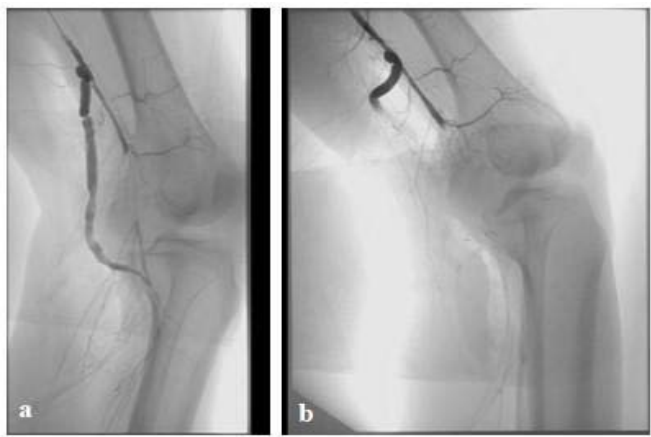

Figure 4: Angiogram showing blood flow. a) Angiogram showing that blood flow is initially maintained below the knee; b) Angiogram showing the interruption of blood flow.

Table 1: Schenck Anatomic Knee Dislocation Classification.

\begin{tabular}{|c|l|}
\hline KD I & Injury to single cruciate and collaterals \\
\hline KD II & Injury to $A C L$ and $P C L$ with intact collaterals \\
\hline KD III-M & Injury to $A C L, P C L$, and $M C L$ \\
\hline KD III-L & Injury to $A C L, P C L$, and $L C L$ \\
\hline KD IV & Injury to $A C L, P C L, M C L$, and $L C L$ \\
\hline KD V & Dislocation and fracture \\
\hline
\end{tabular}

ACL: Anterior Cruciate Ligament; KD: Knee Dislocation; LCL: Lateral Collateral Ligament; MCL: Medial Collateral Ligament; PCL: Posterior Cruciate Ligament.

further worsen the blood flow of the right leg. The patient's knee flexion is currently $140^{\circ}$ and his knee extension is $0^{\circ}$. He can walk using a cane and experiences slight pain.

\section{Discussion/Conclusion}

The rate of vascular injury with knee dislocation is reported to be between $11 \%$ and $19 \%[13,14]$. However, only a few articles have reported cases with a vascular injury that require surgical treatment. According to a report by Parker, one in twenty-five traumatic dislocations of the knee require vascularization [15]. It has also been reported that up to $20 \%$ of patients with vascular injuries require amputation $[12,16]$. Traumatic dislocation of the knee with vascular injury is rare; thus, some orthopedic surgeons may overlook the symptoms of complications owing to inexperience. Therefore, understanding the complications of knee dislocation is important to identify early signs of complications.

Schenck et al. [17] categorized traumatic dislocations of the knee into six distinct types (Table 1). Moatshe et al. [18] analyzed data of 303 patients with knee dislocations and revealed that Knee Dislocation (KD) III-M was the most common type (52.4\%) and KDIII-L was the second most common type (28.1\%) based on the Schenck classification. They also reported that KDIII-L dislocations were significantly associated with popliteal artery injuries. In addition, peroneal nerve injuries were significantly associated with vascular injury [18]. In our case, the patient had ACL, PCL and LCL injuries (KDIII-L), which were further complicated by the development of a sensory disorder of the peroneal nerve region and motor weakness of the right TA and EHL. Thus, classification of dislocation type and neurological evaluation are helpful when assessing vascular injury.

Initially the patient's dorsal artery of the right foot was palpable; 


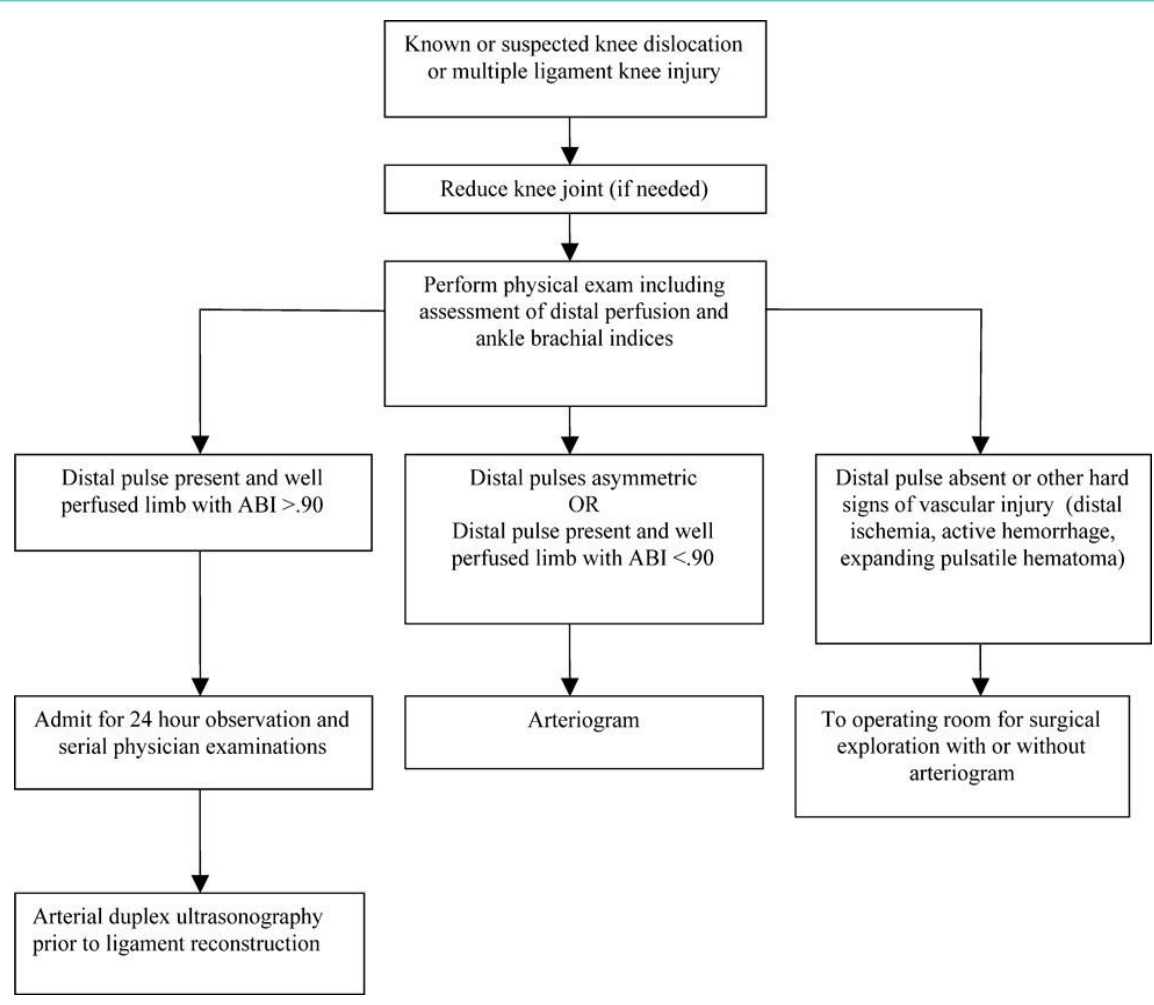

Figure 5: Algorithm for the diagnosis of vascular injury following dislocation of the knee. ABI: Ankle-Brachial Pressure Index.

however, it was not palpable when he was admitted to our hospital. Previous studies have reported that initial palpation of pedal pulses is not sufficient to rule out significant limb-threatening vascular injury [19] because sufficient collateral circulation maintains pedal pulses in the acute stage of complete popliteal rupture [19]. Nicandri et al. recommended an algorithm for the diagnosis of vascular injury with knee dislocation [20] (Figure 5). They insisted that even if the distal artery is palpable or the Ankle-Brachial Pressure Index (ABI) is $>0.9$, a 24-hour observation and serial physical examinations are necessary. They also insisted that angiography should be considered when distal pulses are asymmetric or the $\mathrm{ABI}$ is $<0.9$, and surgical exploration should be considered when the distal pulse is absent or there are other signs of vascular injury. In our case, the patient's dorsal artery of the right foot was not palpable, and CT revealed popliteal injury. Therefore, we conducted angiography before reconstructing the compound ligamentous injuries even though blood flow of the leg worsened during angiography. Through this experience, we learned that once blood flow is obstructed completely, it is difficult for the patient to recover. In addition, reconstruction of ligamentous injuries requires a longer operative time and use of a tourniquet, which can also induce obstruction of the artery. Thus, clinicians must be aware that in some cases, patients with knee dislocation may experience complications such as arterial injuries, which cause severe dysfunction of the knee. Additionally, clinicians must be aware that meticulous and frequent evaluation of vascular injuries is necessary when treating dislocation of the knee.

Even if the blood flow of the limb appears to be maintained, dislocation of the knee can easily induce limb ischemia. Clinicians must pay attention to vascular injuries, particularly when performing surgery for knee dislocation.

\section{Clinical Message}

Knee dislocation requiring revascularization is quite rare. However, it causes severe dysfunction of the knee. Thus, meticulous and frequent evaluation of vascular injuries is necessary for the treatment of knee dislocation.

\section{Consent}

The patient provided informed consent for publication of this report.

\section{References}

1. Arom GA, Yeranosian MG, Petrigliano FA, Terrell RD, McAllister DR. The changing demographics of knee dislocation: a retrospective database review. Clin Orthop Relat Res. 2014; 472: 2609-2614.

2. Brautigan B, Johnson DL. The epidemiology of knee dislocations. Clin Sports Med. 2000; 19: 387-397.

3. Good L, Johnson RJ. The dislocated knee. J Am Acad Orthop Surg. 1995; 3: 284-292.

4. Rihn JA, Cha PS, Groff YJ, Harner CD. The acutely dislocated knee: evaluation and management. J Am Acad Orthop Surg. 2004; 12: 334-346.

5. Stannard JP, Sheils TM, Lopez-Ben RR, McGwin G Jr, Robinson JT, Volgas DA. Vascular injuries in knee dislocations: the role of physical examination in determining the need for arteriography. J Bone Joint Surg Am. 2004; 86: 910.

6. Wascher DC, Dvirnak PC, DeCoster TA. Knee dislocation: initial assessment and implications for treatment. J Orthop Trauma. 1997; 11: 525-529.

7. Peskun CJ, Whelan DB. Outcomes of operative and nonoperative treatment of multiligament knee injuries: an evidence-based review. Sports Med Arthrosc. 2011; 19: 167-173. 
8. Plancher KC, Siliski J. Long-term functional results and complications in patients with knee dislocations. J Knee Surg. 2008; 21: 261-268.

9. Eranki V, Begg C, Wallace B. Outcomes of operatively treated acute knee dislocations. Open Orthop J. 2010; 4: 22-30.

10. Bottoni CR, Liddell TR, Trainor TJ, Freccero DM, Lindell KK. Postoperative range of motion following anterior cruciate ligament reconstruction using autograft hamstrings: a prospective, randomized clinical trial of early versus delayed reconstructions. Am J Sports Med. 2008; 36: 656-662.

11. Mook WR, Miller MD, Diduch DR, Hertel J, Boachie-Adjei Y, Hart JM Multiple-ligament knee injuries: a systematic review of the timing of operative intervention and postoperative rehabilitation. J Bone Joint Surg Br. 2009; 91: 2946-2957.

12. Natsuhara KM, Yeranosian MG, Cohen JR, Wang JC, McAllister DR, Petrigliano FA. What is the frequency of vascular injury after knee dislocation? Clin Orthop Relat Res. 2014; 472: 2615-2620.

13. Robertson A, Nutton RW, Keating JF. Dislocation of the knee. J Bone Joint Surg Br. 2006; 88: 706-711.

14. Khakha RS, Day AC, Gibbs J, Allen S, Hill P, Hull J, et al. Acute surgical management of traumatic knee dislocations-average follow-up of 10 years. Knee. 2016; 23: 267-275.
15. Parker S, Handa A, Deakin M, Sideso M. Knee dislocation and vascular injury: 4 year experience at a UK Major Trauma Centre and vascular hub. Injury. 2016; 47: 752-756.

16. Patterson BM, Agel J, Swiontkowski MF, MacKenzie EJ, Bosse MJ, LEAP Study Group. Knee dislocations with vascular injury: outcomes in the Lower Extremity Assessment Project (LEAP) Study. J Trauma Acute Care Surg. 2007; 63: 855-858.

17. Schenck RC Jr. The dislocated knee. Instr Course Lect. 1994; 43: 127-136.

18. Moatshe G, Dornan GJ, Løken S, Ludvigsen TC, LaPrade RF, Engebretsen L. Demographics and injuries associated with knee dislocation: A prospective review of 303 patients. Orthop J Sports Med. 2017; 5: 2325967117706521.

19. Jones RE, Smith EC, Bone GE. Vascular and orthopedic complications of knee dislocation. Surg Gynecol Obstet. 1979; 149: 554-558.

20. Nicandri GT, Chamberlain AM, Wahl CJ. Practical management of knee dislocations: a selective angiography protocol to detect limb-threatening vascular injuries. Clin J Sport Med. 2009; 19: 125-129. 